\title{
Show Me How You Interact, I Will Tell You What You Think: Exploring the Effect of the Interaction Style on Users' Sensemaking about Correlation and Causation in Data
}

\author{
A'aeshah Alhakamy* \\ University of Tabuk \\ Tabuk, Saudi Arabia \\ aalhakami@ut.edu.sa
}

\author{
Milka Trajkova* \\ Indiana University-Purdue University \\ Indianapolis \\ Indianapolis, Indiana, United States \\ milktraj@iu.edu
}

\author{
Francesco Cafaro* \\ Indiana University-Purdue University \\ Indianapolis \\ Indianapolis, Indiana, United States \\ fcafaro@iu.edu
}

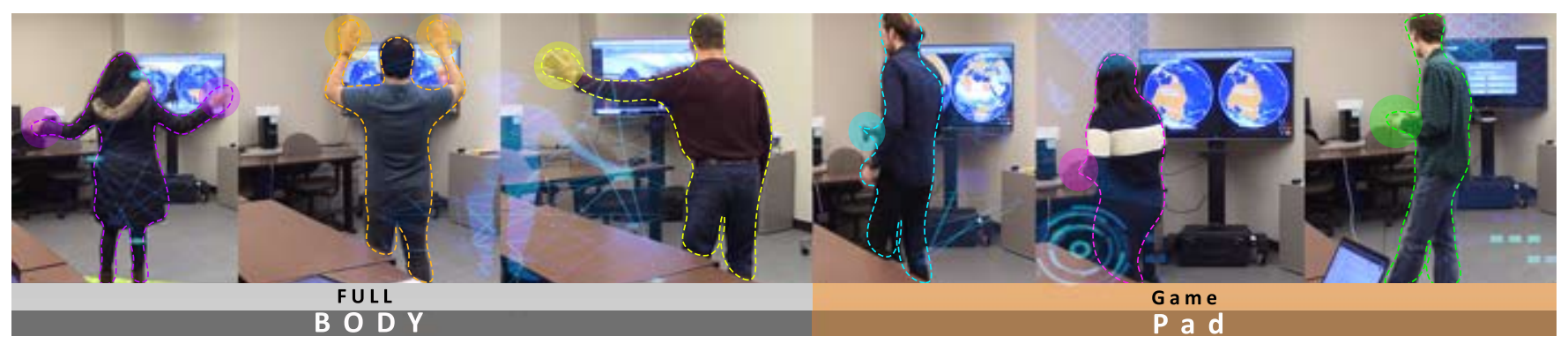

Figure 1: We conducted an in-lab study using two alternative prototypes of an interactive data visualization (two 3D globes representing geo-referenced data on a 65" screen). Participants in the first condition (Full-Body) were able to control the data visualization using mid-air gestures and body movements. Participants in the second condition (Gamepad) used a gamepad to interact with the screen.

\begin{abstract}
Findings from embodied cognition suggest that our whole body (not just our eyes) plays an important role in how we make sense of data when we interact with data visualizations. In this paper, we present the results of a study that explores how different designs of the "interaction" (with a data visualization) alter the way in which people report and discuss correlation and causation in data. We conducted a lab study with two experimental conditions: Full body (participants interacted with a 65" display showing geo-referenced data using gestures and body movements); and, Gamepad (people used a joypad to control the system). Participants tended to agree less with statements that portray correlation and causation in data after using the Gamepad system. Additionally, discourse analysis based on Conceptual Metaphor Theory revealed that users made fewer remarks based on FORCE schemata in Gamepad than in Full-Body.
\end{abstract}

*All authors contributed equally to this research.

This work is licensed under a Creative Commons Attribution International 4.0 License.

DIS '21, June 28-fuly 2, 2021, Virtual Event, USA

(C) 2021 Copyright held by the owner/author(s).

ACM ISBN 978-1-4503-8476-6/21/06.

https://doi.org/10.1145/3461778.3462083

\section{CCS CONCEPTS}

- Human-centered computing $\rightarrow$ User studies; Interaction paradigms; Ubiquitous and mobile computing design and evaluation methods.

\section{KEYWORDS}

Embodied Interaction; Human-Data Interaction; Public Displays; Data Visualization; Informal Learning

\section{ACM Reference Format:}

A'aeshah Alhakamy, Milka Trajkova, and Francesco Cafaro. 2021. Show Me How You Interact, I Will Tell You What You Think: Exploring the Effect of the Interaction Style on Users' Sensemaking about Correlation and Causation in Data. In Designing Interactive Systems Conference 2021 (DIS '21), June 28-fuly 2, 2021, Virtual Event, USA. ACM, New York, NY, USA, 12 pages. https://doi.org/10.1145/3461778.3462083

\section{INTRODUCTION}

In today's world, people produce 2.5 quintillion bytes of data per day -while searching for information on the Internet, reading newspapers, using social media, or listening to debates [2]. Thus, data exploration is no longer limited to the realm of research laboratories, in which scientists define experimental scenarios to validate hypotheses [44]. For example, during the 2020 pandemic, people have spontaneously created hundreds of data visualizations about COVID-19 and posted them on Twitter and other social media [64]. In such a context, being able to make sense of data is a particularly crucial skill, because more and more people have relied on their own data analyses to inform their personal and business decisions 
(for example, whether it is safe enough to go to the beach or to reopen the dining room of their restaurant to customers [64]).

Additionally, as the gathering and visualization of large data sets become central to people, museums have attended to ways of presenting data to visitors ([53]) -including with large displays that people can control with gestures and body movements [15]. Designing installations that facilitate data exploration is, however, still a challenge for Human-Data Interaction [63]. Specifically, museum visitors spend a short amount of time with interactive installations -between 15 seconds and 2 minutes [46, 57] -so we need to identify design strategies that quickly enable people to focus on the data on display [63] and to discuss insights on the data [3].

Within this scenario, this paper focuses on two specific concepts: correlation and causation, which are essential to understanding modern science [12]. Correlation and causation can be difficult to interpret. On the one hand, confounding correlation with causation may lead people to assume causality when there is not. For example, people may erroneously assume that high ice-cream sales cause an increase in the number of deaths by drowning [47], because of the strong correlation between these two variables. On the other hand, the mantra that correlation does not imply causation may conceal how correlation can be a precious hint to causation, and lead people to question the validity of scientific findings (for example, the association between smoking and lung cancer [18]).

Research in data visualization has tackled the problem of crafting visual elements that illustrate causal dependencies: for example, by using animations rather than static charts [35], specific views to facilitate analytical reasoning [60], or side-by-side block diagrams instead of traditional bar charts, treemaps, or mosaic plots [27].

Findings from embodied interaction [22] and embodied learning [34], however, indicate that our body (not just what we see with our eyes) plays a significant role in the way in which we learn and understand abstract concepts. In particular, in cognitive linguistics, Lakoff's and Johnson's [33] Theory of Conceptual Metaphors (CMT) posits that humans gain an understanding of the world using a small set of basic mental patterns, "embodied schemata." These schemata arise from our experiences using our bodies and are acquired at a very young age; they create networks of brain circuits [37] that shape and constraint our thinking and sensemaking. For example, we acquire FORCE-based schemata like COMPULSION after experiencing "being moved by external forces, like wind, water, ..." multiple times [33], or BLOCKAGE when "we encounter obstacles that block or resist our force" [33]. Interestingly, according to Johnson, FORCE schemata always trigger a sequence of causality in our mind (e.g., "the door closes because I, or the wind [...] acted on it to cause it to shut" [33]. Thus, FORCE schemata might be related with people's ability to identify causal patterns in data.

The work that we describe in this paper is grounded on these sociocultural theories. We explored how the users' "interaction" with a data visualization (on a 65" display) contributes to how people make sense of causation and correlation across the datasets on display. We conducted an in-lab study with 20 participants using two different prototypes: Full-Body vs. Gamepad, see Figure 1. We chose these two interaction approaches (based on a Kinect tracking camera or a gamepad) because they are frequently used to control screens in museums and public spaces $[15,50]$.
Our findings indicate that participants tended to agree less with statements that portrayed correlation and causation across the data that they explored after interacting with a gamepad. Additionally, in the Gamepad condition, participants' remarks about the data on display were grounded on FORCE-based schemata four times less frequently than in the Full-Body Condition.

\section{BACKGROUND: EMBODIED COGNITION AND CONCEPTUAL METAPHOR THEORY (CMT)}

Our work is inspired by the theory of Embodied Cognition [66]. According to embodied cognition, our body plays a fundamental role in our cognitive processes: our discoveries happen thanks to the interaction between our body and the surrounding environment [59].

In particular, this work is grounded on Lakoff's and Johnson's Conceptual Metaphor Theory (CMT) [33, 38, 39]. According to CMT, we acquire basic mental patterns -called embodied schemata -at a very early stage of our lives, through our bodily interaction with the world [33]. For example, a baby learns the BALANCE schema when they are able to stand in an erect position [33]. Through time, this schema evolves metaphorically, creating complex networks of mental connections [38] -so we can, for example, apply the idea of BALANCE to abstract domains, such as social justice and architecture. Embodied schemata arise from repeated bodily experiences, for example experiencing an external FORCE, or seeing or feeling physical and visual ATTRIBUTES. They materialize as a meaningful "recurrent pattern, shape, and regularity in our daily body experience" ( [33], p. 29). These mental patterns are integral to our study as they operate as conceptual primitives at the core of our sensemaking. For example, Gentner [26] reported that people typically make sense of electricity using either a WATER-FLOW or a MOVING CROWD model. In the first case, an electric current is understood as water flowing through a pipe; in the latter case, individuals' movement through passageways and small gates. In general, people who make sense of electricity using the WATERFLOW model perform better on battery problems, while those using the MOVING CROWD metaphor excel on resistor problems [26]. Thus, we hypothesized that the way in which we make sense of correlation and causation in a data visualization might depend on the embodied schemata that we use while interacting.

\section{RELATED WORK}

\subsection{Human-Data Interaction (HDI)}

Our work contributes to Human-Data Interaction (HDI) [14, 23]. With HDI, we refer to a research stream investigating how people interact with large sets of data using novel interfaces [14, 23]. For example, the study in Trajkova et al. [63] investigated how different ways of representing the users on a large display affect people's engagement with a gesture-based data visualization.

We want to acknowledge that, as highlighted by Victorelli et al. in their literature review of Human-Data Interaction [65], the term HDI has been used to refer to a broad range of research topics spanning from computer graphics to information science. Mortier et al.'s [48] defined HDI as a multi-disciplinary line of research 
that investigates how people can engage with "personal" data. The reader should notice that our work focuses on data in general, not only on personal data. In other words, we adopted Elmqvist's and Cafaro's definition of HDI, which is different from Mortier's.

Specifically, in the work that we describe in this paper, we are interested in the opportunities afforded by the design of the interaction to facilitate people's sensemaking while they explore large sets of data using interactive data visualizations. As observed in [23], data analysis and visual analytic tools are typically based on traditional windows, icons, menus, pointer (WIMP) interfaces and fail to capitalize on the ways in which the "interaction" itself contributes to people's data sensemaking.

\subsection{Embodied Interaction and Embodied Learning}

Our work is on embodied interaction. The concept of embodied interaction was established through a seminal book by Paul Dourish [22]. According to Dourish, meaning during the interaction is formed through the interplay between people's actions and the social and material context in which they happen. In later work, Hornecker [29] focused on the role of the body in embodied interaction, arguing, for example, that "movement and perception are tightly coupled." In the context of museum installations and large displays, the term full-body Interaction" has been used in a narrower fashion to denote an "input method" that people use when they interact with computer systems using hand gestures and body movements ([15]).

Additionally, our work is inspired by Embodied Learning [34], a sub-field of the learning sciences that seeks to use embodied interaction principles to design technological interventions that facilitate learning. Notable examples include the Situated Multimedia Arts Learning Lab (SmallLab) [11], and Lindgren et al.'s [40] work on mixed-reality environments -which highlighted that students understand psychics concepts better when they asked to embody a meteor rather than play with it on a traditional desktop-based interface [41]. These works, however, require either a personal identification with a virtual object (e.g., being a meteor), culturally-acquired "props," or a 1:1 relationship between each concept and gesture (e.g., making a clockwise gesture to turn a gear clockwise) [40]. Embodied learning has been particularly useful to facilitate the learning of mathematical concepts. In a study with mathematical experts and non-experts, Nathan et al. [49] found that both groups of participants used gestures (and speech) while describing geometric properties -and concluded that promoting gesture production may facilitate mathematical reasoning. DeSutter and Stieff [21] suggested that embodied interaction might facilitate spatial thinking -for example, by re-orienting a scientific visualization showing a molecule depending on the user's left and right movements in front of the screen. Abrahamson and Trninic [1] described an embodied interaction system aimed at facilitating proportional reasoning by asking fifth-grade children to represent a ratio with their hands.

The work in this paper builds upon these findings. It compares how two different interaction modalities (Gamepad vs. Full-Body) affect the user's sense-making around two specific concepts: causation and correlation.

\subsection{Using Conceptual Metaphor Theory for Designing Intuitive Embodied Interactions}

In interaction design [55], Conceptual Metaphor Theory (CMT) has been used by Antle et al. [6] to inform the design of a full-body installation that facilitates the learning of musical concepts: input actions (i.e., gestures and body movements) are mapped into perceptual, auditory feedback. Similarly, Macaranas et al. [43] asked participants to map the material properties of tangible objects (e.g., smooth/rough foam cubes) to abstract concepts (e.g., polite/unpolite). Antle and Wise [7] presented a framework for designing Tangible User Interfaces [32] that facilitate learning. Schuman et al. [58] analyzed the collaborative usage patterns of a touch screen interface showing a data visualization of global ocean temperatures, and found that participants made use of conceptual metaphors through the interaction (in particular, they used spatial words like UP and DOWN to make sense of an increase or decrease in temperature). Roberts et al. [54] described the design of a full-body installation that museum visitors can use to explore how data from the US Census changed over time -and discovered that instrumenting the space with a vertical (rather than horizontal) timeline facilitates temporal reasoning because it better aligns with how we talk about time in English (the past is behind us, the future is in front of us).

Hurtienne and Israel [30] described one of the first attempts to apply CMT to the design of Tangible User Interfaces, which is particularly relevant for the analysis that we describe in this paper. Their noteworthy work lists a table of categories of body experiences (Space, Containment, Multiplicity, Force, Attribute, Process, Surface, and Basic) and the corresponding embodied schemata that belong to each of those categories (e.g., up-down, container, merging, diversion, big-small, matching, substance, etc.) [30]. In later work, Hurtienne et al. [31] described a design approach to use CMT to redesign a touch-screen interface for a sound entertainment system. Although Hurtienne's work does not provide definitions for each schema and does not focus on the design of the interaction with data visualizations, the table in [30] provided the starting point for the coding dictionary that we used in our analysis.

\subsection{Visual Analytics}

Finally, we want to highlight the connection between this work and previous literature on visual analytics [62], a research field that investigates how data visualizations may facilitate analytical reasoning and that "builds on the human mind's ability to understand complex information visually" [62]. For example, the work in [25] reviewed visual analytics tools that allow to visually interpret protein integration and gene expression.

Interestingly for the work that we discuss in this paper, gestures and body movements have been used in visual analytics. In 2007, Ball and North [8] conducted an experiment that compared a mouse-based vs. a full-body approach to navigate a data map on a wall-display -based on the idea that asking people to walk in the interaction space could facilitate their use of spatial memory. Participants expressed a general preference for the physical navigation interaction style, and were faster in accomplishing their data analysis tasks (e.g., zooming in or aggregating data). 
Work on immersive technologies (Virtual, Mixed, and Augmented Reality [45]) for visual analytics has also explored the use of fullbody movements to interact with data visualizations. For example, Cordeil et al. [17] introduced ImAxes, a VR system that allows users to control standard visualizations (e.g., histograms, scatterplots) by walking within a room-sized interaction space while wearing a VR headset, and described usage scenarios for this multi-variate data visualization tool. In a pre-print, Kluge et al. [36] suggested the use of multiple "virtual lenses" to explore 3D immersive visualizations. Betella et al. [9, 10] described virtual and mixed-reality reality systems that enable users to explore the architecture of the human brain using "embodied navigation" and gestures -and found that users performed tasks better when using this VR system, rather than a more traditional desktop interface. Butscher et al. [13] introduced ART, a Mixed-Reality data analysis tool based on a touch-enabled tabletop, and suggested that this approach might facilitate a sense of immersion and collaboration practices.

This line of research focuses on the technical or visual implementation of tools for visual analytics and suggests that embodied interaction may facilitate data analysis tasks. The work that we report in this paper builds upon those ideas, and complements them by exploring the effect of two specific interaction styles (Full-Body vs. Gamepad) on users' sensemaking about correlation and causation when they freely explore a data visualization (rather than having to accomplish a specific data analysis task). Unlike the aforementioned works in immersive technologies for visual analytics, we do not focus on immersive environments, but on a display (a 65" TV) like those that are increasingly used in museums, public spaces, and even people's homes. Additionally, we use the lenses of a theory from cognitive linguistics -Conceptual Metaphor Theory (CMT) -to explore the impact of different interaction styles on the mental patterns that people use to make sense of the data on the screen.

\section{PROBLEM STATEMENT}

The study that we report in this paper explores whether the Interaction Style (Full-Body vs. Gamepad) affects the users' sensemaking about causation and correlation across datasets (when they explore an interactive data visualization). Leveraging on findings from embodied interaction and embodied learning, we hypothesized that asking people to use their body to do different actions (either performing mid-air gestures or body movement, or using a gamepad) would prime them towards using different mental patterns (embodied schemata [38]) and, thus, change the way in which they make sense of correlation and causation in data. Specifically, our study was designed to answer the following research questions:

- (R.Q.1) How does the interaction style (Full-Body vs. Gamepad) affect the users' agreement with statements that imply either correlation or causation across the datasets on display? Significance. We want to explore if the interaction style affects peoples' sensemaking around two important concepts in science: causation and correlation. If so, we can contribute to Human-Data Interaction by providing guidelines for designing interactive installations that facilitate the exploration of correlation and causation patterns in data.
- (R.Q.2) Are any categories of schemata used more/less frequently depending on the Interaction Style?

Significance. According to Conceptual Metaphor Theory, embodied schemata are interconnected in complex networks of mental circuits [37]; thus, if the interaction style primes users towards specific types of schemata and/or away from others, this may activate networks of mental connections that change the way in which we make sense of data. In other words, if the interaction style changes the mental patterns that we detect in the users' language, this can explain differences in how they see causation and correlation in data.

\section{SYSTEM DESIGN \& IMPLEMENTATION}

To test the two Interaction Styles (Full-Body vs. Gamepad), we implemented two variations of a data visualization prototype. Figure 2 provides an overview of the system. Our installation visualizes geo-referenced datasets on two 3D globes. Each prototype consists of two subsystems working simultaneously in real-time: a manager (specific for either the Full-Body or the Gamepad version of the system), and a dataset designer (shared across the two versions of the prototype).

(1) Full body//Gamepad Manager. The manager enables system functionalities such as rotation, zooming, changing datasets, or fly to a certain location. Each condition manager is discussed in detail in the following subsections.

(2) Datasets Designer. Two datasets are visualized on two interactive $3 \mathrm{D}$ globe maps that have realistic earth and atmosphere settings. Each country is represented with different colors mapped on the data values.

\subsection{Full Body Manager}

We used a Microsoft Kinect v.2 camera to implement the Full-Body version of the prototype. Raw video feeds streaming from the depth and color camera sensors are the motion inputs of our system. Image skeletal tracking -which detects one to six players moving within the field of view of the camera -is used in the gesture manager as described below. The 3D positions $(x, y, z)$ of user's joints are identified through Unity packages and custom-made scripts that support the specific functionality of our global system. A user's joints can be tracked over time to detect specific movements, which are then recorded and utilized as a significant gesture to control the data visualization.

We developed multiple gestures to navigate and control the 3D globes, based on the HDI tasks and functionalities list recommended in Trajkova et al. [63]. In the spine movement the globe object is used to overlay the tracked spine mid joint in order to transform and rotate the angle based on the user spine location. The purpose of this functionality is to allow people to explore data at different locations, and to see the data from different perspectives on the globe (to build on spatial reasoning, as done in embodied visual analytic systems, e.g. [8]). Zoom in/out allows users to get a closer look at the data presented on the globe; in particular, we developed a hand movement to get to each country on the globe. This gesture provides a comparing mechanism between two data sets. Jump and Swipe are implemented using a time window to record a certain joint movement (legs for jump, hands for swipe) 


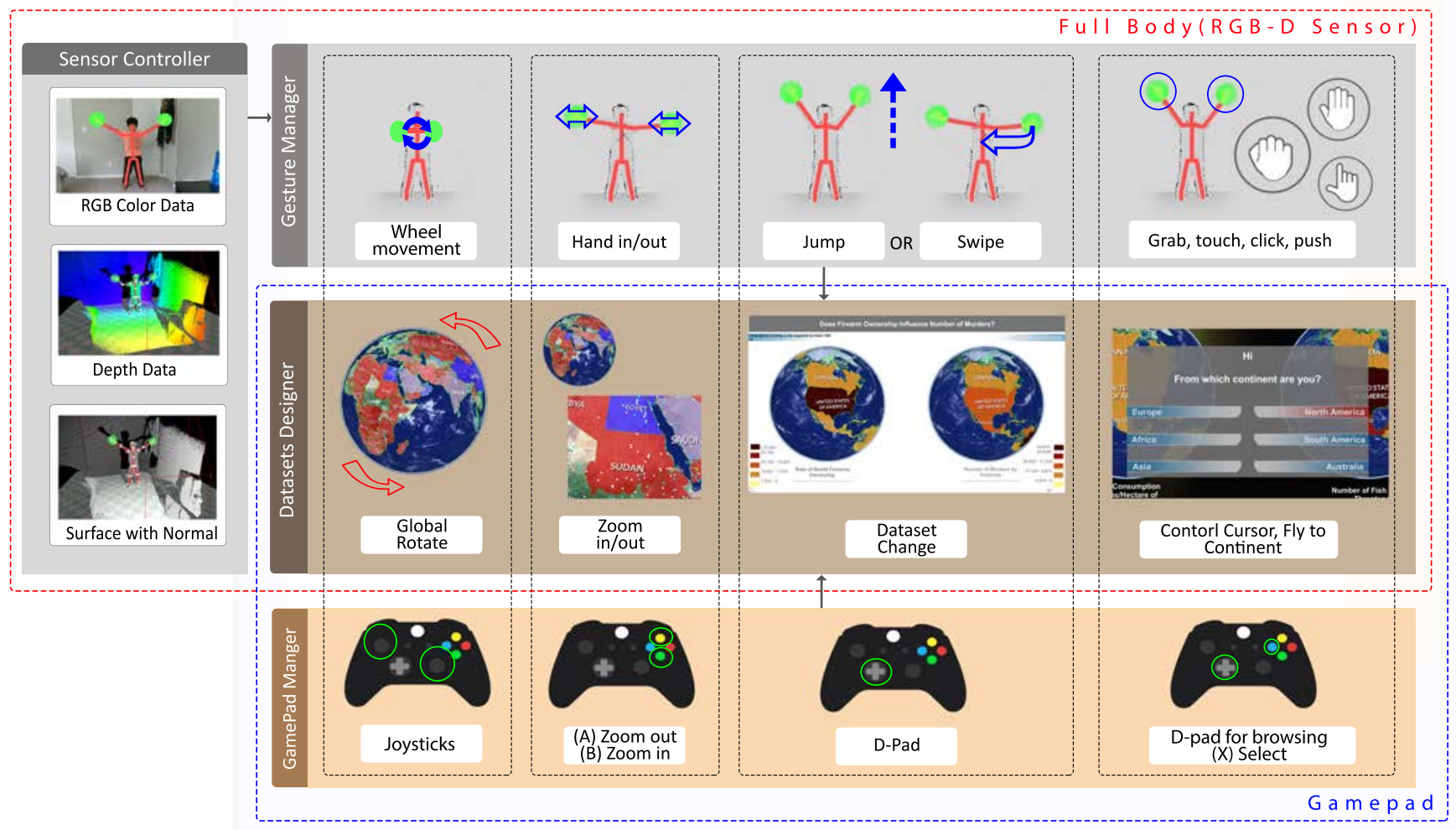

Figure 2: Overview of the system which supports two experimental conditions (A) Full Body(RGB-D Sensors) and (B) Gamepad. The prototype is implemented in a modular way, and consist of (1) Full Body/Gamepad Manger, and (2) Datasets Designer.

for a period of time while a condition is valid. Both gestures allow people to change the data sets presented on both globes. For instance, one of the data sets asks "Does Fertilizer Consumption Influence Number of Threatened Fish Species?" where global data about Fertilizer Consumption is represented on the first globe while the Number of Threatened Fish Species is displayed on the other. On jump/swipe, a different question and data sets are represented, such as "Does Firearm Ownership Influence Number of Murders?". Hand Hold/Grab control the cursor and its functionalities: the user can hand grab, release, and (push)click objects on the screen. This functionality allows to browse menu elements on the screen and provides the ability to explore each data set presented on the globe more in-depth, based on each country or point on the globe.

\subsection{Gamepad Manger}

In the prototype that we implemented to support the Gamepad Interaction Style, the input system does not rely on human gestures, but depends on the input from a gamepad. The idea behind using this gadget instead of a mouse, for instance, was to insert the sense of playing a game and having fun while exploring data instead of being in an office on a regular business day. Therefore, the globe maps are rotated using the joysticks on the gamepad to explore the data from several directions and in different countries. For a closer look at the data value of a specific country or region, the (A), (B) buttons are used for growing and shrinking the globe maps.
D-Pad is used to change the dataset and explore different values on both globes. Finally, the right stick is used for browsing the menus or element on the scene, then use $(\mathrm{X})$ button to select the desired option- see Figure 2.

\subsection{Datasets Designer}

Two 3D globe maps are used to represent the global data of each country in the world. The color of the country's surface represents the data value. The system renders two different datasets that are shown side by side on each globe. The data are uploaded from resource files. The color gradients are based on a value that has been normalized among all datasets to reflect a common color range. There could be up to 20 datasets depicted on the globe maps, together with some thought-provoking, scaffolding questions [28] represented on the top portion of the screen. For our lab-study, we only used 8 of these datasets (4 pairs), along with four questions: (1) "Does firearm ownership influence number of murders?" and (2) "Does refugee number influence unemployment rate?", (3) "Does fertilizer consumption influence number of threatened fish species?", and (4) "Does access to freshwater influence mortality rate?".

\subsection{Hardware and Software Description}

Hardware. The system runs on an Intel ${ }^{\circledR}$ Core $^{\mathrm{TM}}$ i7-4710HQ CPU @ $2.50 \mathrm{GHz} 2501 \mathrm{MHz}, 4$ core(s), 8 logical processor(s) 16.0 GB RAM, 
and NVIDIA GeForce GTX 970 GPU. For the RGB-D sensor (FullBody condition), we used a Microsoft Kinect V2 camera. For the Gamepad condition, we used a Gaming Controller Gamepad Joystick for PC (Windows XP/7/8/10), Steam, Android, and PS3.

Software. Unity3D engine was used as the main platform for the entire system and the collective of tools and packages are installed based on the requirement of each task. For body tracking and gesture support, we used the Kinect for Windows Software Development Kit (SDK) v. 2.0 to develop the system with gestural interaction in $\mathrm{C} \#, \mathrm{C}++$, and Java. Also, a world map bundle was utilized in our system after modification.

\section{METHODOLOGY}

To assess the impact of the Interaction Style (Full-Body vs. Gamepad) on how participants make sense of Correlation and Causation in data, we conducted a mixed-method in-lab experimental study. We adopted a between-study design: participants interacted either with the Full-Body or the Gamepad prototype -we did not expose them to both versions of the system, because we wanted to isolate the effect of each Interaction Style. Participants interacted with the system one at a time. We randomized the order of the experimental conditions; this resulted in 20 study sessions, 11 for the Full-Body condition, and 9 for the Gamepad condition. All study sessions were video recorded and transcribed.

\subsection{Participants}

Twenty people (11 identified themselves as males and 9 as females) participated in this experiment. We recruited them using emails and mailing lists at a large university campus. They belonged to a wide range of age groups, from 21 to 49 years old, with most of them $(\mathrm{N}=13)$ in the 21-29 age range. They had different levels of education, including High school degrees $(\mathrm{N}=1)$, Associate $(\mathrm{N}=1)$, Bachelor $(\mathrm{N}=7)$, Master $(\mathrm{N}=6)$, Professional $(\mathrm{N}=1)$, and Doctorate degrees $(\mathrm{N}=1)$. Participants belonged to a range of races, including Asian $(\mathrm{N}=12)$, white $(\mathrm{N}=6)$, Native Hawaiian or Pacific Islander $(\mathrm{N}=1)$, and Other $(\mathrm{N}=1)$.

\subsection{Experimental Procedure}

Each participant was greeted by a moderator and invited to review and sign a consent document. The experimental sessions followed a testing protocol script and consisted of the following three steps: a pre-test questionnaire, the interaction with the prototype, and a post-test questionnaire.

Pre-Test. Participants were asked to fill out a Qualtrics survey on an iPad that asked how much they agreed with two statements related to correlation and causation. Such two statements were repeated for all four pairs of datasets that people were then going to see on the screen during the interaction phase: (1) Fertilizer Consumption and Number of Threatened Fish Species; (2) Firearm Ownership and the Number of Murders; (3) Number of Refugees and the Unemployment Rate; (4) Access to Freshwater and Mortality Rate. Specifically, participants were asked:

"On a scale from 1 (strongly disagree) to 5 (strongly agree), how much do you agree with each of the following statements?

(1) Fertilizer consumption is correlated with the number of threatened fish species;
(2) Increased fertilizer consumption causes an increase in the number of threatened fish species.

The order in which the pairs of datasets were presented in the survey was randomized to eliminate question order bias (e.g., one participant saw the statements about Fertilizer Consumption and Number of Threatened Fish Species first, another started with Gun Ownership and Number of Murders, and so on). The pre-test survey was followed by four demographic questions concerning participants' age, race, gender, and educational level.

Interaction. Following the pre-test, the users were asked to freely use the interactive installation and were given simple instructions that described how to interact. Participants were given one to two minutes to get acquainted with the interactive screen, and then asked to think aloud and answer the following two questions: 1) Can you tell me what you see on the screen?; 2) Can you describe the data on the screen? Participants in the Full-Body condition interacted using mid-air gestures and body movements; participants in the Gamepad condition used the gamepad to navigate the data visualization.

Post-Test. After interacting with the system, users were given a post-test questionnaire that asked them to rate the same questions as in the pre-test. The order in which such statements were presented was the same order in which each participant had seen them in the pre-test.

\subsection{Embodied Schemata Code Analysis}

In preparation for the analysis, five researchers collaboratively looked at the transcripts of the think-aloud remarks that participants made while interacting with the installation, in order to identify the idea units [4] (i.e., we were not coding for key words, but looking for full sentences that explained the same concepts) pertinent to the data on display. We want to highlight that, because our analysis focuses on data sensemaking, we conducted this analysis only on the idea units in which participants made remarks about the data on display, and excluded remarks related to the system's usability. We then used the catalog of Embodied Schemata provided by Hurtienne et al. [30] as the initial dictionary for coding those idea units. We performed this type of linguistics analysis because previous work in embodied interaction has shown that people make remarks based on embodied schemata and cognitive metaphors when they explore interactive data visualization (e.g., $[54,58])$. Due to a lack of definitions and keywords describing each schema, however, we took an inductive approach to better understand how to categorize and group our data. The following sections describe the rounds of coding and data analysis.

Initial Round. Five researchers individually looked at the list of idea units that we identified as pertinent to the data on display from participants' interviews, and coded them (when they deemed appropriate) using the catalog of Embodied Schemata reported by Hurtienne et al. [30]. Each researcher initially coded 1/5 of the list. While doing that, the researchers highlighted the most significant keywords (in each idea unit) that suggested them to apply a specific embodied schema.

The reader should notice that coding for embodied schemata means that the researchers used linguistic analysis to look into participant's mind, i.e. to interpret which mental patterns (embodied 
schemata) participants used to ground their sensemaking while making remarks about the data on display. Because embodied schemata are polysemic [33], the same idea unit can be grounded on multiple (not only one) embodied schemata.

Second Round. In order to cross-validate the coding, each researcher individually looked at the portion of the list that they did not directly code ( $4 / 5$ of the list) in round one, and indicated whether they agreed or not on the code(s) applied to each quote. In case of disagreement, they listed the code they would have used, and/or the alternative keywords they would have used to identify such codes.

Final Round. The researchers worked synchronously (in virtual Zoom meetings, because the analysis was conducted during the COVID-19 pandemic) to discuss each idea unit. The goal was to resolve disagreement and both embodied schemata and keywords. At the end of this process, the team of researchers agreed on $98 \%$ of the overall coding for the idea units (there was a disagreement on 3 of the 144 idea units, which were then excluded from the statistical analysis).

\subsection{Data Preparation for Statistical Analysis}

To prepare the data for statistical analysis on the impact of the Interaction Style on the agreement score that participants assigned to correlation and causation statements (R.Q.1), we cleaned the data and removed one instance of response bias, i.e. one participant in the Full-Body Interaction style put the same Likert scale response for every question in the pre and post tests. The final number of participants came down from 20 to 19 participants. We then computed the average agreement score in each of the pre-test and post-test scores. With average agreement score we mean that, in each test (pre and post tests), we considered the average of the four scores that participants assigned to each of the four correlation statements, and the average of the four scores assigned to the causation statements. We used the average because we were interested in the overall effect of Interaction Style on participants' sensemaking on causation and correlation, not to assess individual differences across the datasets on display.

To analyze if the different Interaction Type (Full-Body vs. Gamepad) resulted in people's using different combinations of categories of embodied schemata (R.Q.2), we computed the frequencies of each of the categories of schemata (e.g., SPACE, CONTAINMENT, MULTIPLICITY, etc. - see Table 1) in each Interaction Type. We based our analysis on the category of schemata (rather than on individual embodied schemata) to provide more generalizable results (the specific embodied schemata that people use may be dependent on our application scenario or data visualization).

\section{RESULTS}

\subsection{R.Q.1: Impact of the Interaction Style on Correlation and Causation}

The study design included three variables: two within-subject factors, (1) the Test Time (pre-test vs. post-test), because each participant had to fill out a survey at different times (before and after the interaction), (2) the Question Type (correlation vs. causation), as each participant had to rate statements about both causation and and correlation; and one between-subject factor, (3) The Interaction Style (Full-Body vs. Gamepad) because each participant interacted with the data visualization using either gestures and body movements, or a gamepad. We wanted to analyze the interaction of these three independent variables on the participant's average agreement score (the dependent variable) with causation and correlation statements in the pre- and post-tests.

In order to do that, we conducted a three-way mixed ANOVA, with the test time (pre-test vs. post-test) and the question type (correlation vs. causation) as within-subjects factors, and the interaction style (Full-Body vs. Gamepad) as a between-subjects factor. The scores were normally distributed, as assessed by Shapiro-Wilk's test $(p>.05)$. There was homogeneity of variances, as assessed by Levene's test for equality of variances $(p>.05)$. There was not a statistically significant three-way interaction between test time, question type, and interaction style, $F(1,16)=0.268, p=0.612$. We continued the analysis because there was a statistically significant twoway interaction between Interaction Style (Full-Body vs. Gamepad) and Test Time (pre-test vs. post-test), $F(1,16)=7.743, p=0.013$. In other words, asking participants to interact using either full-body gestures or a gamepad altered the difference in the average agreement scores that users assigned during pre vs. post-tests to statements that portray correlation and causation in the data that they explored. The reader should notice that, at this point in the analysis, for each interaction style, the score is the average of those on causation and correlation statements, and the same results could be obtained using a two-way mixed ANOVA that ignores Question Type. Contrary to our original expectation, there was no statistically significant simple main effect of Test Time (pre-test vs. post-test) in the Full-Body interaction style, as detected by one-way repeated measure ANOVA, $F(1,18)=0.396, p=0.537$. There was, instead, a statistically significant simple main effect of Test Time in the Gamepad interaction style, $F(1,19)=21.274, p<0.0001$. Specifically, the average score decreased from the pre-test $(X=3.275, S D=0.466)$ to the post-test $(X=2.6123, S D=0.716)$-see Figure 3 .

\subsection{R.Q.2: Impact of the Interaction Style on Categories of Schemata}

After detecting that the Interaction Style affected the post-test scores, we wanted to dig more into the users' mental patterns to explore what could explain this difference. As we described in the methodology section, this portion of the analysis was structured in two parts: (1) coding participants' remarks during the interaction; and, (2) statistical analysis to identify differences across Interaction Types.

7.2.1 Coding. Among all participants' remarks during the interaction, we identified 144 idea units in which users described the data on display. Table 1 lists the embodied schemata (among those listed by Hurtienne [30]) that we used to code those idea units, the Categories of Body Experience [33] that we used to group them, and the keywords that we inductively identified from the transcripts as indicative of each schema. We want to highlight a lack of previous work on how to detect embodied schemata in the remarks that people make when they interact with a data visualization. Thus, keywords and examples in 


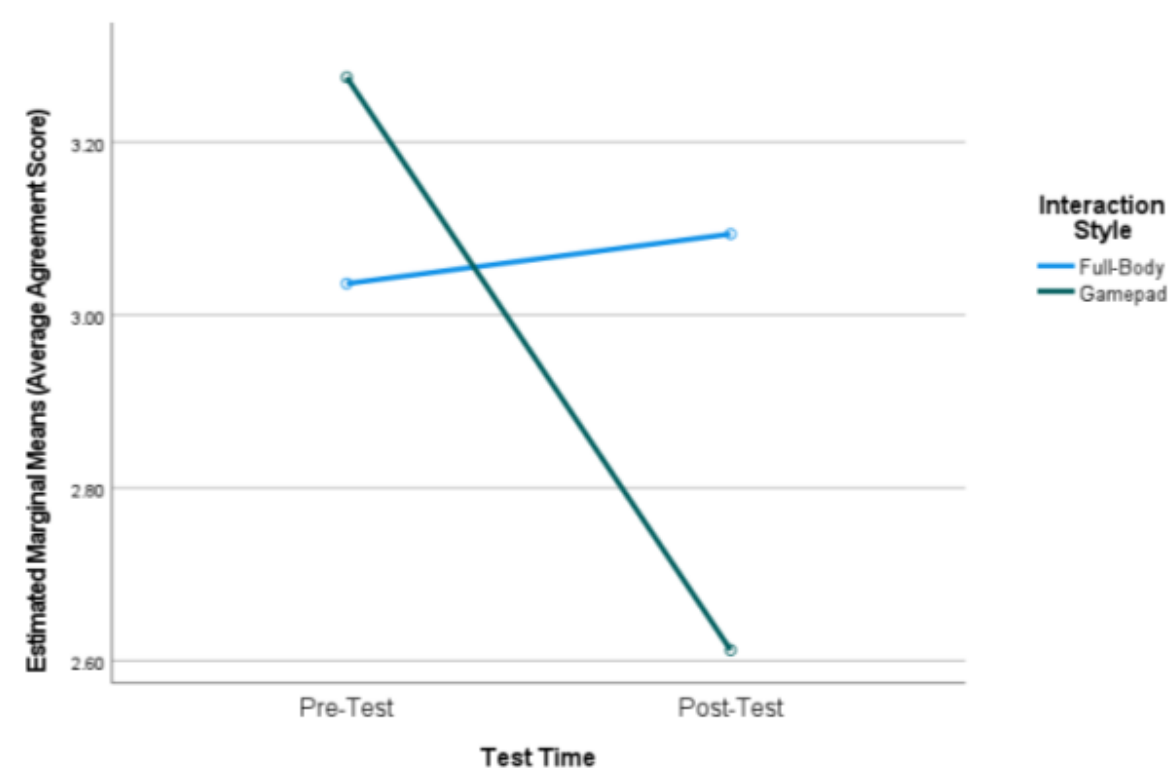

Figure 3: A plot of the estimated marginal means for the average agreement scores that participants assigned during pre-test vs. post-test in the two Interaction Styles (Full-Body vs. Gamepad)

7.2.2 Statistical Analysis. At this point, we had enough data to assess if there was an interaction between the Interaction Type and the Categories of Embodied Schemata that we coded (see Table 1), i.e. if the frequency of the categories of schemata on which people grounded their remarks about the data on display changed when using different Interaction Styles. As before, the variable Interaction Style has two groups (Full-Body vs. Tangible), while the Category of Schemata variable has six groups (SPACE, CONTAINMENT, MULTIPLICITY, FORCE, ATTRIBUTE, SURFACE -see Table 1). We did not use a (simpler) chi-square test because the number of groups would have required many z-test comparisons during the posthoc analysis, and because many idea units were coded with multiple (not only one) schemata (chi-squares are not good for multiple-choice problems). Rather, we performed a two-way log-linear analysis.

The likelihood ratio for the association between Interaction Type and Categories of Embodied Schemata was statistically significant, $\chi^{2}(5)=15.639, p=0.008$. In other words, interacting using a gamepad vs. with gestures and body movements affects the categories of embodied schemata that participants use in their remarks.

Regarding the specific categories of schemata, the log-linear model detected a statistically significant difference in the use of FORCE schemata between the two Interaction Styles. Specifically, the odds ratio of participants reasoning using FORCE schemata when they interacted using the Gamepad was $20 \%$ of the odds of participants using FORCE schemata when they interacted using their Full-Body -see Table 2.

The reader should notice that $20 \%$ comes from $e^{-1.609}$. In other words, participants made remarks based on FORCE schemata four times more frequently while interacting with gestures and body movements than when they used a Gamepad.

There were other statistically significant differences within Interaction Styles (for example, consistently with literature on embodied interaction [6], SPACE schemata were overall the most frequently used mental patterns across the two experimental conditions), but these go beyond the scope of our research question.

\section{DISCUSSION}

\subsection{Interaction Design and Data Sesemaking: Impact of the Interaction on Users' Sensemaking about Data}

Our results indicate that the Interaction Style (Gamepad vs. FullBody) influences how users make sense of correlation and causation: the difference in people's agreement with statements that portray correlation or causation between pre and post tests (Test Time) depended on the Interaction Style. Thus, different interaction styles do not only provide an engaging way to interact with large displays [46]; rather, they alter the way in which we make sense of data when we explore data visualizations. This complements findings that embodied interaction approaches may facilitate tasks in visual analytics (e.g., the "embodied navigation" in [8]).

We want to acknowledge that this paper does not intend to provide a definitive answer on which interaction style is "better" to support "reasoning" about or "learning" of correlation or causation: the focus is on sensemaking in general, while the evaluation of reasoning and learning should be investigated in future work. This could be a particularly fruitful line of research, because an increasing body of research demonstrates that hand gestures play an important role in scientific reasoning in classrooms. For example, multiple studies report that students and teachers use gestures when talking about science in formal learning settings (e.g., [19, 20, 51, 56]). Singer, Radinsky, and Goldman [61] found that the use of gestures in a 6th grade science class demonstrated advancement in the group's understanding of plate tectonics. Chen 
Table 1: Catalog of some Embodied Schemata [30] including the dictionary keywords extracted from the idea units from the participants' remarks during our experiment.

\begin{tabular}{|c|c|c|c|}
\hline $\begin{array}{l}\text { Category } \\
\text { of Schemata }\end{array}$ & $\begin{array}{l}\text { Embodied } \\
\text { Schemata }\end{array}$ & Dictionary - Keyword & Quote Example \\
\hline \multirow[t]{3}{*}{ SPACE } & UP-DOWN & $\begin{array}{l}\text { Greater, higher, high, low, bottom, top, } \\
\text { lower, highest, little }\end{array}$ & $\begin{array}{l}\text { "I get that is because of the consumption of fertilizers } \\
\text { higher in China..." }\end{array}$ \\
\hline & SCALE & $\begin{array}{l}\text { Number, rate, bad, worse, better, cleaner, } \\
\text { lot, more, weighted scale, amount, } \\
\text { many, large, percentage }\end{array}$ & $\begin{array}{l}\text { "But the mortality rate of course is worse in poor coun- } \\
\text { tries..." }\end{array}$ \\
\hline & LOCATION & $\begin{array}{l}\text { Globe, places, side, particular, parts, } \\
\text { world, space, over there, At the coun- } \\
\text { try, particular continent, Africa, Aus- } \\
\text { tralia, Europe, America, Brazil, Mex- } \\
\text { ico, Italy, Turkey, Finland, China, In- } \\
\text { dia, Nigeria, Argentina, United States, } \\
\text { Venezuela, Croatia, Bolivia, Columbia, } \\
\text { France, Spain, Algeria, Syria, Iran }\end{array}$ & $\begin{array}{l}\text { "it looks like access to water is pretty consistent across } \\
\text { the globe..." }\end{array}$ \\
\hline \multirow[t]{3}{*}{ CONTAINMENT } & CONTAINER & $\begin{array}{l}\text { lake, countries, in, in the country, on } \\
\text { the screen, mark on the }\end{array}$ & $\begin{array}{l}\text { "They probably have lot of Lake fishes because the ac- } \\
\text { cess to water is not done much." }\end{array}$ \\
\hline & IN-OUT & In & $\begin{array}{l}\text { "They have all locked right in from Turkey and yeah, } \\
\text { Finland as well." }\end{array}$ \\
\hline & FULL-EMPTY & Not noticed & "So I did not noticed it." \\
\hline \multirow[t]{2}{*}{ MULTIPLICITY } & COUNT-MASS & Counted & $\begin{array}{l}\text { "The refugees are obviously unauthorized at [a] particu- } \\
\text { lar continent and they are being still counted" }\end{array}$ \\
\hline & LINKAGE & Engaging, connected & "It is entirely getting connected with each other. Right," \\
\hline \multirow[t]{2}{*}{ FORCE } & DIVERSION & Doubt, overlooked, misleading & $\begin{array}{l}\text { "There is no water around the area, there [are] no work- } \\
\text { ers [that] still live [there]. It is like making a mark on } \\
\text { the map. It looks misleading to me because I cannot } \\
\text { make sense out of where in India." }\end{array}$ \\
\hline & BLOCKAGE & $\begin{array}{l}\text { Cannot make sense, struggling, not be } \\
\text { able to understand, not exactly sure, } \\
\text { more clear, be able to }\end{array}$ & $\begin{array}{l}\text { "Like for example, Brazil has very little firearm posses- } \\
\text { sion, but it looks like the highest firearm on the side } \\
\text { rates. But yeah, Brazil, Venezuela and Mexico all stand } \\
\text { out pretty clearly there. I'm not exactly sure what the } \\
\text { significance of Bolivia and Columbia data is like." }\end{array}$ \\
\hline \multirow[t]{2}{*}{ ATTRIBUTE } & $\begin{array}{l}\text { DARK- } \\
\text { BRIGHT }\end{array}$ & Color, white (black) & "All these data identified as zero in orange color" \\
\hline & BIG-SMALL & Large & $\begin{array}{l}\text { "Syria has a large number of refugees but doesn't have } \\
\text { any information on employment." }\end{array}$ \\
\hline SURFACE & MATCHING & $\begin{array}{l}\text { Seems, indicate, identified, mapping, } \\
\text { correlated, same, correlation, related, } \\
\text { opposite, like, both, compared, compare, } \\
\text { equal, differences, assumed, thought, ap- } \\
\text { pear, As a, influence, both maps, con- } \\
\text { sistent, looks like, different, relative to, } \\
\text { relationship }\end{array}$ & $\begin{array}{l}\text { "I can see there is no correlation between access to } \\
\text { water and mortality rate..." }\end{array}$ \\
\hline
\end{tabular}

Table 2: We detected a statistically significant difference in participants' use of FORCE based schemata between the two experimental conditions (Gamepad and Full-Body).

\begin{tabular}{|l|l|c|c|c|c|}
\hline Int. Style & Category & $(\lambda)$ & $Z$ & $p$ & $\%$ of baseline \\
\hline Full-Body & FORCE & 2.303 & 7.281 & & (baseline) \\
\hline Gamepad & FORCE & -1.609 & -2.078 & 0.038 & $20 \%$ \\
\hline
\end{tabular}

and Herbst [16] report that, when limited information is given, students compensated those limitations by engaging with gestural expressions. Thus, we believe that specific gestures may play a particularly important role in building reasoning about data -even beyond formal learning settings.

\subsection{Embodied Schemata and Data Sensemaking: Activating Networks of Mental Circuits to Facilitate Data Sensemaking}

Because of its grounding on sociocultural theories of cognition, the study in this paper provides insights into why different interaction styles alter sensemaking. In particular, we want to stress that we did not include generic remarks on the usability of the system (e.g., users' comments on the gamepad, or on the accuracy of the gestures and body movements recognition) in our analysis for R.Q.2. We took 
this approach because one could expect from previous literature on embodied interaction that users will comment on the exertion (or fatigue) required to perform gestures and body movements (e.g., [42]), or on characteristics of the movement itself (e.g., having to BALANCE ourselves [5] after jumping). An example of the quotes that we did not include in the analysis is: $(\mathrm{P} 15)$ So I can rotate it when I do that. [...] That's kinda cool.

Rather, the idea units that we considered were deemed related to the data on display by a team of five researchers who coded the transcripts. For example, in Table 1, the quote on DIVERSION suggests that the user is reasoning about properties of the data representation (something we did not see happening in the Joystick condition), while the remark coded as BLOCKAGE indicates that the user is trying to relate the data on display with her prior knowledge on the topic -a type of personal insights similar to those observed by Roberts et al. [52] and that Trajkova et al. see as particularly relevant for designing Human-Data Interaction [63].

This trend suggests that the schemata that are triggered while performing gestures and body movements may propagate in the user's mind in a way that activates similar, related mental patterns (i.e., FORCE schemata activated by movements can, in turn, activate other FORCE schemata that facilitate making sense of the data visualization). This is consistent with Lakoff's theory that embodied schemata form an actual network of mental circuits [37]. These results also resonate with work in embodied interaction that has found -through language analysis -that people use embodied schemata and cognitive metaphors when they describe the data on display (e.g., [54, 58]). We believe this trend indicates the need for future, fundamental research investigating which embodied schemata aid data sensemaking, reasoning, and learning. We also need to identify the most suitable interaction modalities to activate those schemata.

\subsection{Implications for Human-Data Interaction}

For example, our results show that interacting with a gamepad (rather than with hand gestures and body movements) reduces the users' agreement with statements that portray correlation and causation across the dataset on display. This finding has implications for designing interactive displays that allow people to compare and analyze large sets of data (Human-Data Interaction [14, 23]). In particular, it suggests that Gamepad may be a suitable interaction style when we want to prevent people from mistakenly seeing causation across the datasets on display.

\subsection{Relationship between FORCE Schemata and Causality}

Our results indicate that interacting using a Gamepad resulted in fewer remarks based on FORCE mental patterns (when participants described the data on display during their interaction with the visualization) compared to the remarks that people made while interacting with hand gestures and body movements.

We already mentioned how the FORCE schemata activated during the interaction might, in turn, activate other FORCE schemata on which people ground their conversation (and sensemaking) about the data on display. Additionally, as we mentioned in the Introduction, CMT suggests a deeper interplay between FORCE schemata and causal reasoning. Specifically, FORCE schemata always involve a "sequence of causality" [24]. For example, Johnson observes that "the door closes because I, or the wind, or a spring mechanism, acted on it to cause it to shut" [33] (p. 44). The networks of mental connections [38] between FORCE schemata and causality are activated pre-conceptually in our brain, without even noticing.

Our findings suggest that the "sequence of causality" intrinsic in FORCE schemata may prime people towards seeing causality (and correlation) across the dataset on display. This could explain why, in the Gamepad Interaction Style, people used fewer FORCE schemata, made fewer remarks grounded on FORCE schemata when they described the data on display and, in turn, agreed less with causation and correlation statements after interacting with the system. Future work should further investigate this phenomenon by comparing additional interaction styles (e.g., a more office-based scenario with keyboard and mouse; systems that respond to static/iconic gestures vs. more dynamic full body movements; etc.), data visualizations (different from the globe-based interface that we used), display sizes (e.g., wall displays, mobile phones, etc.), and data sets.

\subsection{Application Scenarios}

We decided to conduct this study in-lab because we needed to isolate as much as possible the variable that we manipulated (i.e., the Interaction Style) from other environmental factors. The lab setting allowed to expose all participants to the same data visualization, and to make them interact in the same space (in front of a 65" display) and with the same number of people in the room (two moderators). Future studies should bring these prototypes in situ (e.g., at museums, galleries, or other public spaces), to investigate the impact of the social space on our findings.

\section{LIMITATIONS}

In the study that we presented in this paper, we used two gaming devices: a gamepad, and a Microsoft Kinect. We want to acknowledge that people may not be confident in using the gamepad if they do not regularly play games; likewise, they may not be familiar with mid-air gestures and body movements if they never play games using Kinect. Although we believe that we mitigated this problem by randomizing the assignment of participants to experimental conditions, this factor might have influenced the usability of the prototypes (and this work did not investigate the relationship between usability and data sensemaking).

A second limitation that we want to acknowledge is that we did not assess if participants had a correct understanding of causation and correlation before interacting with our prototypes. In this case, we believe that we may have mitigated this problem by randomizing participants, because participants with clearer (or less clear) understanding of causation and correlation were randomly split between the two experimental conditions. This means, however, that this work could not directly assess the impact of prior knowledge on data sensemaking, nor the learning that might have occurred thanks to the interaction with our prototypes.

\section{CONCLUSION}

In this paper, we presented an in-lab study that investigated the role of two Interaction Styles (Gamepad vs. Full-Body) on the way 
in which users make sense of causation and correlation in data. Our results indicate that these two interaction styles affected how people make sense of causation and correlation in different ways: participants in the Full-Body conditions made more remarks based on FORCE schemata while they explored the data visualization; participants in the Gamepad condition used FORCE schemata significantly less, and tended to agree less with statements that portray causation and correlation across datasets on display after they interacted with the system.

Future work should investigate if different interaction styles can prime people towards using specific schemata, and the role of the social space (e.g., what happens in public spaces like museums).

\section{ACKNOWLEDGMENTS}

This material is based upon work supported by the National Science Foundation under Grant No. 1848898 (CHS:Eager:Aiding Reasoning about Correlation and Causation). Special thanks to the graduate students who helped with the data collection or coding: Sanika Vedak, Rashmi Mallappa, and Sreekanth R. Kankara.

\section{REFERENCES}

[1] Dor Abrahamson and Dragan Trninic. 2011. Toward an Embodied-Interaction Design Framework for Mathematical Concepts. In Proceedings of the 10th International Conference on Interaction Design and Children (Ann Arbor, Michigan) (IDC '11). Association for Computing Machinery, New York, NY, USA, 1-10. https://doi.org/10.1145/1999030.1999031

[2] Irfan Ahmad and Irfan Ahmad. 2020. How Much Data Is Generated Every Minute? [Infographic]. https://www.socialmediatoday.com/news/how-much-data-isgenerated-every-minute-infographic-1/525692/

[3] Sue Allen. 2003. Looking for learning in visitor talk: A methodological exploration. In Learning conversations in museums. Routledge, 265-309.

[4] Richard C Anderson, Ralph E Reynolds, Diane L Schallert, and Ernest T Goetz. 1977. Frameworks for comprehending discourse. American educational research journal 14, 4 (1977), 367-381.

[5] Alissa N Antle, Greg Corness, and Allen Bevans. 2011. Springboard: Designing image schema based embodied interaction for an abstract domain. In Whole body interaction. Springer, 7-18.

[6] Alissa N Antle, Milena Droumeva, and Greg Corness. 2008. Playing with The Sound Maker : Do Embodied Metaphors Help Children Learn? Proceedings of the 7th international conference on Interaction design and children IDC 08 (2008), 178-185. http://portal.acm.org.www.library.gatech.edu:2048/citation.cfm?id= 1463689.1463754

[7] Alissa N Antle and Alyssa F Wise. 2013. Getting down to details: Using theories of cognition and learning to inform tangible user interface design. Interacting with Computers 25, 1 (2013), 1-20.

[8] Robert Ball and Chris North. 2007. Realizing embodied interaction for visual analytics through large displays. Computers \& Graphics 31, 3 (2007), 380-400. https://doi.org/10.1016/j.cag.2007.01.029

[9] Alberto Betella, Enrique Martínez Bueno, Wipawee Kongsantad, Riccardo Zucca Xerxes D. Arsiwalla, Pedro Omedas, and Paul F. M. J. Verschure. 2014. Understanding Large Network Datasets through Embodied Interaction in Virtual Reality. In Proceedings of the 2014 Virtual Reality International Conference (Laval, France) (VRIC '14). Association for Computing Machinery, New York, NY, USA, Article 23, 7 pages. https://doi.org/10.1145/2617841.2620711

[10] Alberto Betella, Rodrigo Carvalho, Jesus Sanchez-Palencia, Ulysses Bernardet and Paul F. M. J. Verschure. 2012. Embodied Interaction with Complex Neuronal Data in Mixed-Reality. In Proceedings of the 2012 Virtual Reality International Conference (Laval, France) (VRIC '12). Association for Computing Machinery, New York, NY, USA, Article 3, 8 pages. https://doi.org/10.1145/2331714.2331718

[11] David Birchfield, Ellen Campana, Sarah Hatton, Mina Johnson-Glenberg, Aisling Kelliher, Loren Olson, Christopher Martinez, Philippos Savvides, Lisa Tolentino, and Sibel Uysal. 2009. Embodied and Mediated Learning in SMAL Lab: A Student-centered Mixed-reality Environment. In ACM SIGGRAPH 2009 Emerging Technologies (SIGGRAPH '09). ACM, New York, NY, USA, 9:1--9:1. https://doi.org/10.1145/1597956.1597965

[12] Mario Bunge. 1997. Mechanism and explanation. Philosophy of the social sciences 27, 4 (1997), 410-465.

[13] Simon Butscher, Sebastian Hubenschmid, Jens Müller, Johannes Fuchs, and Harald Reiterer. 2018. Clusters, Trends, and Outliers: How Immersive Technologies Can
Facilitate the Collaborative Analysis of Multidimensional Data. Association for Computing Machinery, New York, NY, USA, 1-12. https://doi.org/10.1145/ 3173574.3173664

[14] Francesco Cafaro. 2012. Using embodied allegories to design gesture suites for human-data interaction. In Proceedings of the 2012 ACM Conference on Ubiquitous Computing - UbiComp '12. ACM Press, New York, New York, USA, 560. https: //doi.org/10.1145/2370216.2370309

[15] Francesco Cafaro, Alessandro Panella, Leilah Lyons, Jessica Roberts, and Josh Radinsky. 2013. I see you there!: developing identity-preserving embodied interaction for museum exhibits. In Proceedings of the 2013 ACM annual conference on Human factors in computing systems. ACM, New York, 1911-1920. https://doi.org/10.1145/2466110.2466252

[16] Chia-Ling Chen and Patricio Herbst. 2013. The interplay among gestures, discourse, and diagrams in students' geometrical reasoning. Educational Studies in Mathematics 83, 2 (2013), 285-307.

[17] Maxime Cordeil, Andrew Cunningham, Tim Dwyer, Bruce H Thomas, and Kim Marriott. 2017. Imaxes: Immersive axes as embodied affordances for interactive multivariate data visualisation. In Proceedings of the 30 th annual ACM symposium on user interface software and technology. 71-83.

[18] Jerome Cornfield, William Haenszel, E Cuyler Hammond, Abraham M Lilienfeld, Michael B Shimkin, and Ernst L Wynder. 1959. Smoking and lung cancer: recent evidence and a discussion of some questions. Fournal of the National Cancer institute 22, 1 (1959), 173-203.

[19] Elaine M Crowder. 1996. Gestures at work in sense-making science talk. The fournal of the Learning Sciences 5, 3 (1996), 173-208.

[20] Elaine M Crowder and Denis Newman. 1993. Telling what they know: The role of gesture and language in children's science explanations. Pragmatics \& Cognition 1, 2 (1993), 341-376.

[21] D DeSutter and M Stieff. 2017. Teaching students to think spatially through embodied actions: Design principles for learning environments in science, technology, engineering, and mathematics. Cognitive research: principles and implications 2, 1 (2017), 1-20.

[22] Paul Dourish. 2001. Where the action is: the foundations of embodied interaction. MIT Press, Cambridge, MA, USA.

[23] Niklas Elmqvist. 2011. Embodied human-data interaction. In ACM CHI 2011 Workshop "Embodied Interaction: Theory and Practice in HCI". ACM, USA, 104107.

[24] Charles Forceville. 2016. The FORCE and BALANCE schemas in JOURNEY metaphor animations. Multimodality and performance (2016), 8-22.

[25] Nils Gehlenborg, Seán I O'donoghue, Nitin S Baliga, Alexander Goesmann, Matthew A Hibbs, Hiroaki Kitano, Oliver Kohlbacher, Heiko Neuweger, Reinhard Schneider, Dan Tenenbaum, et al. 2010. Visualization of omics data for systems biology. Nature methods 7, 3 (2010), S56-S68.

[26] Dedre Gentner and Donald R Gentner. 2014. Flowing waters or teeming crowds: Mental models of electricity. In Mental models. Psychology Press, 107-138.

[27] Vladimir Guchev, Massimo Mecella, and Giuseppe Santucci. 2012. Design Guidelines for Correlated Quantitative Data Visualizations. In Proceedings of the International Working Conference on Advanced Visual Interfaces (Capri Island, Italy) (AVI '12). Association for Computing Machinery, New York, NY, USA, 761-764. https://doi.org/10.1145/2254556.2254703

[28] Derek Holton and David Clarke. 2006. Scaffolding and metacognition. International journal of mathematical education in science and technology 37, 2 (2006), 127-143.

[29] Eva Hornecker. 2011. The role of physicality in tangible and embodied interactions. interactions 18, 2 (2011), 19-23.

[30] Jörn Hurtienne and Johann Habakuk Israel. 2007. Image schemas and their metaphorical extensions: intuitive patterns for tangible interaction. In Proceedings of the 1st international conference on Tangible and embedded interaction. 127-134.

[31] Jörn Hurtienne, Kerstin Klöckner, Sarah Diefenbach, Claudia Nass, and Andreas Maier. 2015. Designing with image schemas: resolving the tension between innovation, inclusion and intuitive use. Interacting with Computers 27, 3 (2015), 235-255.

[32] Hiroshi Ishii and Brygg Ullmer. 1997. Tangible bits: towards seamless interfaces between people, bits and atoms. In Proceedings of the ACM SIGCHI Conference on Human factors in computing systems. 234-241.

[33] Mark Johnson. 1987. The Body in the Mind: The Bodily Basis of Meaning, Imagination, and Reason. 1987. Chicago: $U$ of Chicago $P$ (1987).

[34] Mina C Johnson-Glenberg, David A Birchfield, Lisa Tolentino, and Tatyana Koziupa. 2014. Collaborative embodied learning in mixed reality motion-capture environments: Two science studies. Fournal of Educational Psychology 106, 1 (2014), 86.

[35] Srinivas R Kadaba, Farooq Ullah Khan, Eshwar Pittampalli, Ashok N Rudrapatna, Ganapathy Subramanian Sundaram, Subramanian Vasudevan, and Yunsong Yang. 2007. Multiple mode data communication system and method and forward and/or reverse link control channel structure. US Patent 7,158,504.

[36] Sven Kluge, Stefan Gladisch, Uwe Freiherr von Lukas, Oliver Staadt, and Christian Tominski. 2019. Virtual Lenses as Embodied Tools for Immersive Analytics. arXiv preprint arXiv:1911.10044 (2019). 
[37] George Lakoff. 2009. The neural theory of metaphor. Available at SSRN 1437794 (2009).

[38] George Lakoff and Mark Johnson. 2008. Metaphors we live by. University of Chicago press, USA.

[39] George Lakoff, Mark Johnson, et al. 1999. Philosophy in the flesh: The embodied mind and its challenge to western thought. Vol. 640. Basic books New York.

[40] Robb Lindgren and Mina Johnson-Glenberg. 2013. Emboldened by Embodiment: Six Precepts for Research on Embodied Learning and Mixed Reality. Educational Researcher 42, 8 (2013), 445-452. https://doi.org/10.3102/0013189X13511661

[41] Robb Lindgren and J Michael Moshell. 2011. Supporting children's learning with body-based metaphors in a mixed reality environment. In Proceedings of the 10th International Conference on Interaction Design and Children. 177-180.

[42] Leilah Lyons, Brian Slattery, Priscilla Jimenez, Brenda Lopez, and Tom Moher 2012. Don't forget about the sweat: effortful embodied interaction in support of learning. In Proceedings of the Sixth International Conference on Tangible, Embedded and Embodied Interaction. 77-84.

[43] Anna Macaranas, Alissa N. Antle, and Bernhard E. Riecke. 2012. Bridging the Gap: Attribute and Spatial Metaphors for Tangible Interface Design. Association for Computing Machinery, New York, NY, USA, 161-168. https://doi.org/10. $1145 / 2148131.2148166$

[44] Viktor Mayer-Schönberger and Kenneth Cukier. 2013. Big data: A revolution that will transform how we live, work, and think. Houghton Mifflin Harcourt.

[45] Paul Milgram, Haruo Takemura, Akira Utsumi, and Fumio Kishino. 1995. Augmented reality: A class of displays on the reality-virtuality continuum. In Telemanipulator and telepresence technologies, Vol. 2351. International Society for Optics and Photonics, 282-292.

[46] Swati Mishra and Francesco Cafaro. 2018. Full Body Interaction Beyond Fun: Engaging Museum Visitors in Human-Data Interaction. In Proceedings of the Twelfth International Conference on Tangible, Embedded, and Embodied Interaction (TEI '18). ACM, New York, NY, USA, 313-319. https://doi.org/10.1145/3173225. 3173291

[47] Leslie M Moore. 1996. The basic practice of statistics.

[48] Richard Mortier, Hamed Haddadi, Tristan Henderson, Derek McAuley, and Jon Crowcroft. 2014. Human-data interaction: The human face of the data-driven society. Available at SSRN 2508051 000, 00, Article 00 (2014), 00 pages.

[49] Mitchell J Nathan, Kelsey E Schenck, Rebecca Vinsonhaler, Joseph E Michaelis Michael I Swart, and Candace Walkington. 2020. Embodied geometric reasoning: Dynamic gestures during intuition, insight, and proof. Journal of Educational Psychology (2020).

[50] Pai Chet Ng, James She, Kang Eun Jeon, and Matthias Baldauf. 2017. When Smart Devices Interact With Pervasive Screens: A Survey. ACM Trans. Multimedia Comput. Commun. Appl. 13, 4, Article 55 (Aug. 2017), 23 pages. https://doi.org/ $10.1145 / 3115933$

[51] Chris Rasmussen, Michelle Stephan, and Karen Allen. 2004. Classroom mathematical practices and gesturing. The fournal of Mathematical Behavior 23, 3 (2004), 301-323.
[52] Jessica Roberts, Francesco Cafaro, Raymond Kang, Kristen Vogt, Leilah Lyons, and Josh Radinsky. 2013. That's Me and That's You: Museum visitors' perspectivetaking around an embodied interaction data map display. (2013).

[53] Jessica Roberts and Leilah Lyons. 2020. Examining spontaneous perspective taking and fluid self-to-data relationships in informal open-ended data exploration. fournal of the Learning Sciences 29, 1 (2020), 32-56.

[54] Jessica Roberts, Leilah Lyons, Francesco Cafaro, and Rebecca Eydt. 2014. Interpreting Data from Within: Supporting Humandata Interaction in Museum Exhibits Through Perspective Taking. In Proceedings of the 2014 Conference on Interaction Design and Children (IDC '14). ACM, New York, NY, USA, 7-16. https://doi.org/10.1145/2593968.2593974

[55] Yvonne Rogers, Helen Sharp, and Jenny Preece. 2011. Interaction design: beyond human-computer interaction. John Wiley \& Sons.

[56] Wolff-Michael Roth. 2001. Gestures: Their role in teaching and learning. Review of educational research 71, 3 (2001), 365-392.

[57] Cody Sandifer. 1997. Time-based behaviors at an interactive science museum: Exploring the differences between weekday/weekend and family/nonfamily visitors. Science Education 81, 6 (1997), 689-701.

[58] Carrie Schuman, Kathryn A Stofer, Lisa Anthony, Hannah Neff, Peter Chang, Nikita Soni, Alice Darrow, Annie Luc, Amanda Morales, Jeremy Alexandre, et al. 2020. Ocean data visualization on a touchtable demonstrates group content learning, science practices use, and potential embodied cognition. Research in Science Education (2020), 1-13.

[59] Lawrence Shapiro. 2019. Embodied cognition. Routledge.

[60] Yedendra Babu Shrinivasan and Jarke J van Wijk. 2008. Supporting the analytical reasoning process in information visualization. In Proceedings of the SIGCHI conference on human factors in computing systems. 1237-1246.

[61] Melissa Singer, Joshua Radinsky, and Susan R Goldman. 2008. The role of gesture in meaning construction. Discourse Processes 45, 4-5 (2008), 365-386.

[62] J. J. Thomas and K. A. Cook. 2006. A visual analytics agenda. IEEE Computer Graphics and Applications 26, 1 (2006), 10-13. https://doi.org/10.1109/MCG.2006.5

[63] Milka Trajkova, A'aeshah Alhakamy, Francesco Cafaro, Rashmi Mallappa, and Sreekanth R. Kankara. 2020. Move Your Body: Engaging Museum Visitors with Human-Data Interaction. In Proceedings of the 2020 CHI Conference on Human Factors in Computing Systems (Honolulu, HI, USA) (CHI '20). Association for Computing Machinery, New York, NY, USA, 1-13. https://doi.org/10.1145/ 3313831.3376186

[64] Milka Trajkova, Francesco Cafaro, Sanika Vedak, Rashmi Mallappa, Sreekanth R Kankara, et al. 2020. Exploring Casual COVID-19 Data Visualizations on Twitter: Topics and Challenges. In Informatics, Vol. 7. Multidisciplinary Digital Publishing Institute, 35.

[65] Eliane Zambon Victorelli, Julio Cesar Dos Reis, Heiko Hornung, and Alysson Bolognesi Prado. 2019. Understanding human-data interaction: Literature review and recommendations for design. International fournal of HumanComputer Studies 000, 00, Article 00 (2019), 00 pages.

[66] Margaret Wilson. 2002. Six views of embodied cognition. Psychonomic bulletin \& review 9, 4 (2002), 625-636. https://doi.org/10.3758/BF03196322 